\title{
Preparative Methods for Nanoanalysis of Materials with Focused Ion Beam Instruments
}

\author{
Philipp M. Nellen*, Victor Callegari, and Urs Sennhauser
}

\begin{abstract}
Over the past 15 years, with materials research moving into sub-micrometer-dimensions, focused ion beam instruments (FIB) have proven to be a versatile tool to prepare samples for nanoanalysis. Especially advanced dual-beam FIBs, i.e. FIBs with a combination of an ion and electron column, take advantage of their particular features for imaging and preparation. This article discusses the principle of ion beam sample interaction to demonstrate how samples are prepared and what kind of possible sample damage and artifacts may occur. Typical FIB instrumentation is also addressed. Finally progress in FIB preparative methods for nanoanalysis of materials and also the common pitfalls to be avoided are discussed.
\end{abstract}

Keywords: Focused ion beam · Nanoanalysis · Preparative tool

\section{Introduction}

Focused ion beam instruments (FIB) have been used in the semiconductor industry and research for chip development, fabrication and process control, failure analysis, and modification since their commercialization in the 1980 s. The reason is that scanning ion beams allow sputtering and modification of conducting and insulating structures of size $10 \mathrm{~nm}$ to $100 \mu \mathrm{m}$. Using reactive gases, which are injected through gas needles some tens of micrometers above the sample, material sputtering can be selectively enhanced up to a factor of 20 for metal and oxide layers, and arbitrary shapes, lines, and dots of metals or oxides can be deposited at specific locations. This avoids time- and cost-intensive repetitive redesign and photolithographic manufacturing in the development phase, by first localizing faults and then correcting design errors directly on the prototype chip. A

\footnotetext{
${ }^{*}$ Correspondence: Dr. P.M. Nellen

Empa, Swiss Federal Laboratories for Materials Testing and Research Electronics/Metrology Laboratory

Überlandstrasse 129

$\mathrm{CH}-8600$ Dübendorf

Tel.: +4144823 4353

Fax: +41 448234054

E-Mail: philipp.nellen@empa.ch
}

typical FIB session allows such tasks to be done within a few hours. General overviews of FIB technology and applications can be found in refs [1][2].

Selective structuring, however, is also useful in materials research [3-5]. Most prominent FIB preparation techniques for nanoanalysis are cross-sectioning [6] and transmission electron microscope (TEM) lamella preparation [7], including target preparation [8]. Newer processes use precise three-dimensional structuring [9][10] to fabricate, amongst others, micro- and nano-pillars for mechanical characterization at the nanoscale, fine periodic line- and dot-structures for deformation-tracking in testing [11], scanning-probe microscope tips for improved resolution like atomic-, electric, magnetic force microscope (AFM, EFM, MFM) [12-15], electrochemical probes [12][16] and scanning near field optical microscope (SNOM) [17], contacts for nano-wires [18] and -tubes for electrical and thermal characterization [19], and irradiated dots on semiconductors as nucleation sites for quantum dots [20].

This article starts with an overview of the interaction of the ion probe with the sample and FIB instrumentation as far as it is required to understand sample preparation. Typical as well as new preparative methods for nanoanalysis with FIB are addressed, starting from sophisticated TEM lamella preparations towards techniques which make extensive use of the capability of FIB for precise structuring. Sample preparation including sample damage, artifacts, and common pitfalls are discussed.

\section{Ion Probe Sample Interaction}

In FIB, accelerated ions (typically between $20-50 \mathrm{kV}$ and most often $\mathrm{Ga}^{+}$) are focused on the sample and interact in two ways (electronic and nuclear stopping) with the target atoms while slowing down and ultimately stopping (Fig. 1) [21]. Their interaction is different from electrons, because their mass is several ten thousand times heavier. At high energies ( $>100 \mathrm{keV})$, inelastic collisions between electrons of the sample and ions are predominant (electronic stopping); the electron clouds of the sample atoms are excited. The dominant process at lower energies, however, is nuclear stopping, i.e. the repulsive interaction is Coulombic with the nuclei screened by the electron clouds. This leads to elastic collisions of the incident ions with the target atoms and can be modeled as a binary collision cascade by Monte Carlo simulations. Ions are backscattered or penetrate the sample to a certain depth. Sample atoms recoil (resulting in damage inside the sample) and the sample is physically sputtered, which is the main process used for structuring. The range of ions in amorphous matter at the given energies is $10-30 \mathrm{~nm}$. Stopping is very localized and thus leads to damage of the material structure (crystal lattice), finally leading to amorphization, and $\mathrm{Ga}$ contamination. Thus imaging by detection of secondary ions, electrons or backscattered electrons, even at low ion currents, always modifies the surface atomic layers. At higher currents the sputtering yield increases with typical values at perpendicular 
incidence, e.g. for silicon, of 2.1 atoms/ion $\left(0.26 \mu \mathrm{m}^{3} / \mathrm{nC}\right)$. The sputter yield strongly depends on the angle of incidence with a material-dependent maximum, often around $70-85^{\circ}$, and on the atomic number and mass of the sample atoms or molecules. Electron emission depends on the atomic number $\mathrm{Z}$ of the sample and on the orientation of polycrystals (ions undergo channeling). This can be used to gather information about composition (Z-contrast imaging), grain size, boundaries, and orientation.

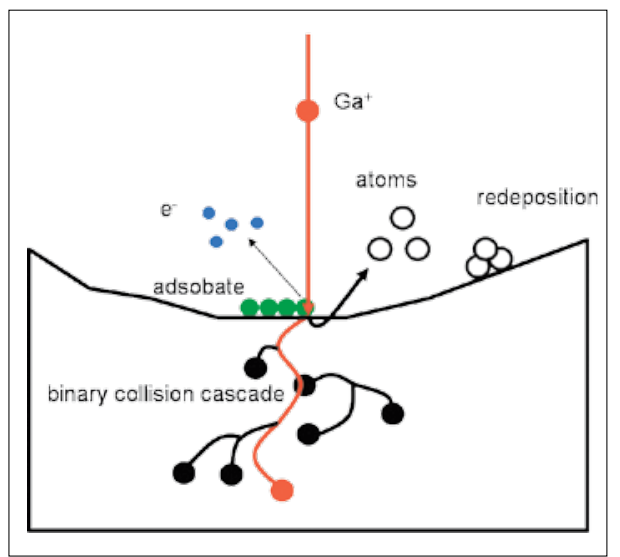

Fig. 1. Interaction of ions with the sample: Elastic collision with atoms of the sample, sputtering of atoms and redeposition, gas enhanced sputtering or materials deposition with adsorbed reactive gases, and secondary electrons and ions emission.

By reactive gas injection the sputter yield can be increased between 2-20 times [1][2]. Gas flows over the sample, adsorbs on the surface and upon ion (or electron) bombardment is activated to (selectively) enhance the sputter yield or to etch and deposit various materials. Common gases used are iodine and chlorine compounds for etching metals, fluorine compounds for etching dielectrics, and water vapor for selectively etching carbon as well as platinum and tungsten compounds for metal deposition and siloxane with water vapor for depositing dielectrics. The reaction rate is governed, amongst others, by the number of gas molecules or ions supplied, whichever is exhausted first.

\section{FIB Instrumentation}

FIB is often referred to as SIM (scanning ion beam microscope) implicating that it operates similarly to a SEM (scanning electron microscope), i.e. the focused ion beam is scanned over the sample while either emitted secondary ions, or electrons, or backscattered electrons are detected with the appropriate detectors (Fig. 2). Gallium $(\mathrm{Ga})$ is a preferred source because of its low melting point $\left(29.6{ }^{\circ} \mathrm{C}\right)$ and low vapor pressure $\left(<<10^{-8}\right.$ Torr at room temperature $)$. At the emission tip liquid $\mathrm{Ga}$ forms a Taylor cone and ions are extracted via an accelerating voltage between 20 to $50 \mathrm{kV}$ and then collimated and focused with electrostatic lenses. The ion beam current density, typically a Holtsmark distribution [22], is often approximated by a Gaussian distribution. The current range is between $1 \mathrm{pA}$ and $30 \mathrm{nA}$ and the ion beam has a spatial resolution of $10 \mathrm{~nm}$ for low currents. The position of the ion beam is controlled by a beam deflection unit which allows arbitrary patterns to be written on the surface.

The reactive gases are injected with the help of nozzles pointing towards the sample with outlets as close as 100-200 $\mu \mathrm{m}$. During gas flow, the pressure of $10^{-6}$ mbar in the vacuum chamber is slightly increased.

An optical microscope or video camera makes it easier to pre-position the sample with the help of a goniometer with five or six axis of freedom. Sophisticated software allows efficient work for specific applications like pre-shaping of TEM lamellae, chip navigation, drift correction, and others.

In a dual-beam FIB an electron column is oriented relative to the ion column at a fixed angle which allows imaging of the milled area during or after milling and with which also electron deposition of materials is possible. A FIB instrument, e.g. a FEI dual-beam Strata 235 DB has typically an ion column of $30 \mathrm{kV}$ with a current range of $1 \mathrm{pA}-20 \mathrm{nA}$, current densities larger than $10 \mathrm{~A} / \mathrm{cm}^{2}$, and a resolution of $7 \mathrm{~nm}$ at $1 \mathrm{pA}$. The electron column of $0.2-30 \mathrm{kV}$ has a relative angle to the ion column of $52^{\circ}$ and a resolution of $3 \mathrm{~nm}$ at $1 \mathrm{keV}$.

\section{Preparative Methods for Nanoanalysis}

FIB is a versatile tool for materials scientists. However, recently also biologists have started to explore the possibilities of FIB preparation including cryogenic prepa-

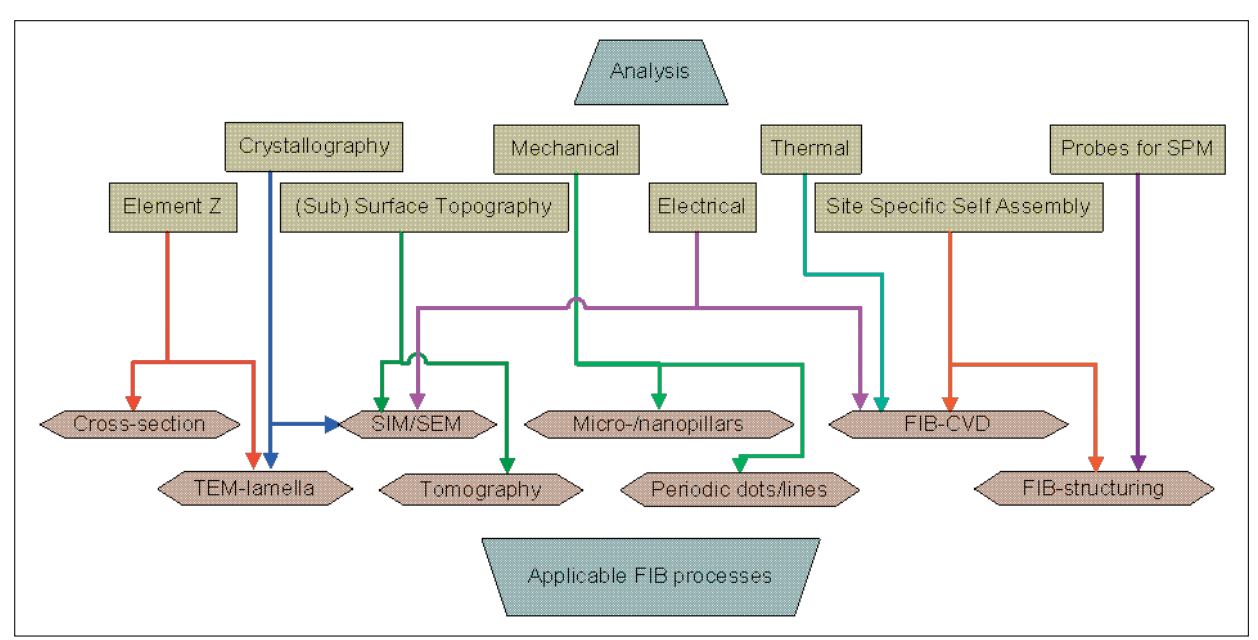

Fig. 3. Selected analytical methods and corresponding preparative FIB processes

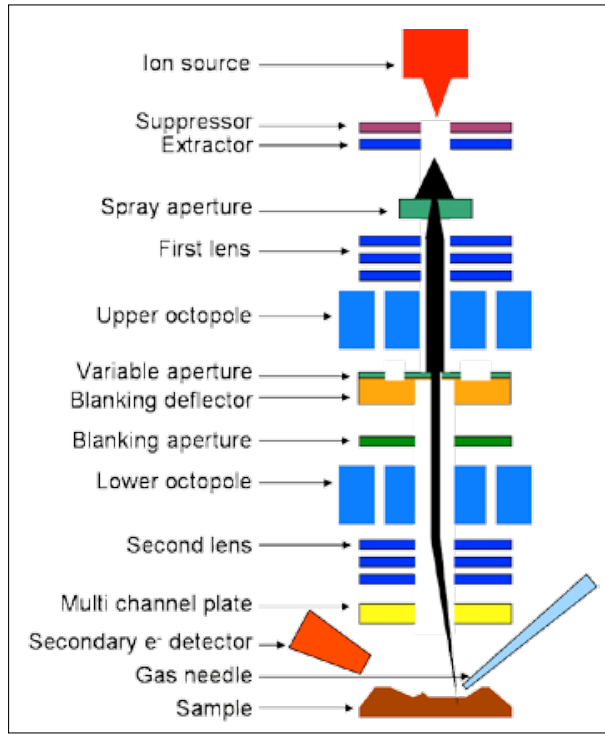

Fig. 2. Simplified scheme of ion beam optics

ration of tissues and unicellular organisms [23][24]. Fig. 3 shows an overview of selected analytical methods and the corresponding preparative techniques with FIB.

\subsection{Cross-sections and 3D-imaging}

Mechanical polishing is often used for metallographic investigations. Yet it fails when materials of different hardnesses are involved, because it smears out the soft material and often leaves lubricating oil making it difficult to see details. Fig. 4a shows a secondary electron image of an ion beam scan of a mechanically polished lead-free solder sample $\left(\mathrm{Sn}_{3.8} \mathrm{Ag}_{0.7} \mathrm{Cu}\right)$ surrounded by a bronze ( $8 \% \mathrm{Sn})$ substrate. No details are visible. In contrast, a similar sample was (after mechanical polishing) continuously polished with $\mathrm{Ga}^{+}$ions at $30 \mathrm{keV}$, $3 \mathrm{nA}$ and any remaining residues including native oxides on the surface were removed. Channeling and Z-contrast were found to be best when imaging is done at $500 \mathrm{pA}$ (Fig. 4b). On the image grain sizes (distribution), form, boundaries, and orientation, two intermetallic particles, and the CuSn- 


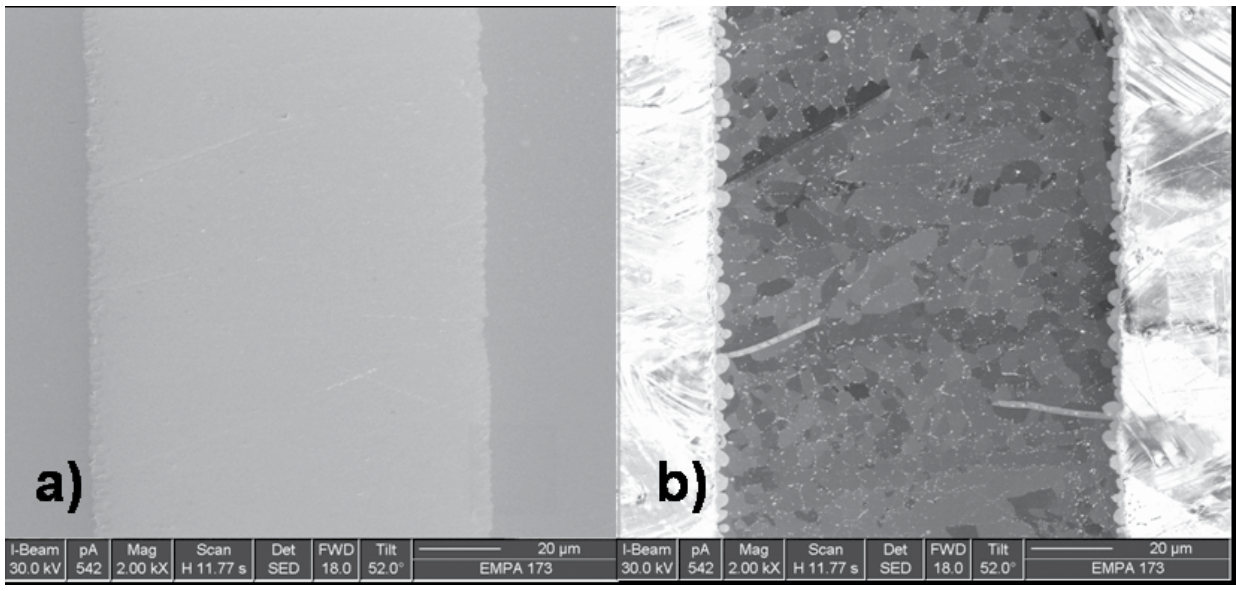

Fig. 4: Secondary electron image of an ion beam scan (perpendicular to surface) of a lead-free solder sample ( $\left.\mathrm{Sn}_{3.8} \mathrm{Ag}_{0.7} \mathrm{Cu}\right)$; a) mechanically polished and b) FIB-polished

phase between bronze and solder can be recognized.

Also cross-sections, triangle-shaped trenches with a flat surface perpendicular to the sample surface, are often used for analysis with either Z-contrast-imaging for differentiation of compound materials, channeling-imaging for grain analysis, or for pore analysis inside the sample. Fig. 5 gives an example of $\mathrm{Z}$-contrast imaging of a cross section of a glass solder (CuZnTiZr).

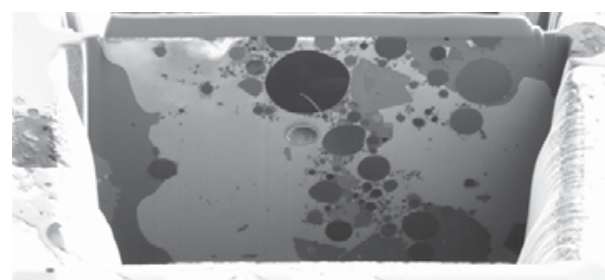

Fig. 5. Ion beam scan of a cross-section of glass solder (CuZnTiZr) unveiling Z-contrast of different grain materials

Imaging with either secondary electrons or ions is sensitive to topography. By alternate sputtering of a thin slice at the surface of a cross section and imaging the newly exposed material a series of 2D images are produced which can be merged and analyzed with dedicated software to a 3D image of inner structures like pores, channels, materials and grain distributions, etc. This technique is often referred to as FIB-tomography [25][26]. A slice thickness as thin as $20 \mathrm{~nm}$ can be achieved. Alternatively, by milling layer by layer downwards into the sample and imaging each exposed surface a series of images is obtained, which can also be analyzed with 3D software. While the first technique limits the resolution to about $20 \mathrm{~nm}$ per slice (accuracy of ion beam shift), the second one can uncover layers of $1 \mathrm{~nm}$ thickness (low current milling) and is fast, but interpretation will be more difficult as soon as materials with large differences in sputter yield are involved. In addition, structural changes and $\mathrm{Ga}$ deposition have to be considered. In Fig. 6a the top layer of a chip with metal lines of width $2 \mu \mathrm{m}$ is ion scanned and simultaneously imaged. Each ion scan with $300 \mathrm{pA}$ and $48.2 \mu$ s dwell time removes roughly $3 \mathrm{~nm}$ of material. By repetitive scanning (thus sputtering) and simultaneously imaging each time, a 3Dimage of the metallization is reconstructed with a total depth of $1.5 \mu \mathrm{m}$ at a magnification of $2000 \times$ (Fig. 6b).

\subsection{TEM Lamella Preparation}

A FIB system is an ideal tool for rapid in situ TEM-lamella preparation [27-29]. A typical lamella size is $20 \mu \mathrm{m} \times 10 \mu \mathrm{m}$ $\times 100 \mathrm{~nm}$. Crystallographic and elemental analysis over most of the area of a lamella is possible once it is properly fabricated. Compared to traditional preparation methods, e.g. microtome, for certain materials lamella preparation is expedited or even made possible at all only with FIB. For example, combinations of hard and weak compounds will not smear out as they do by mechanical thinning.

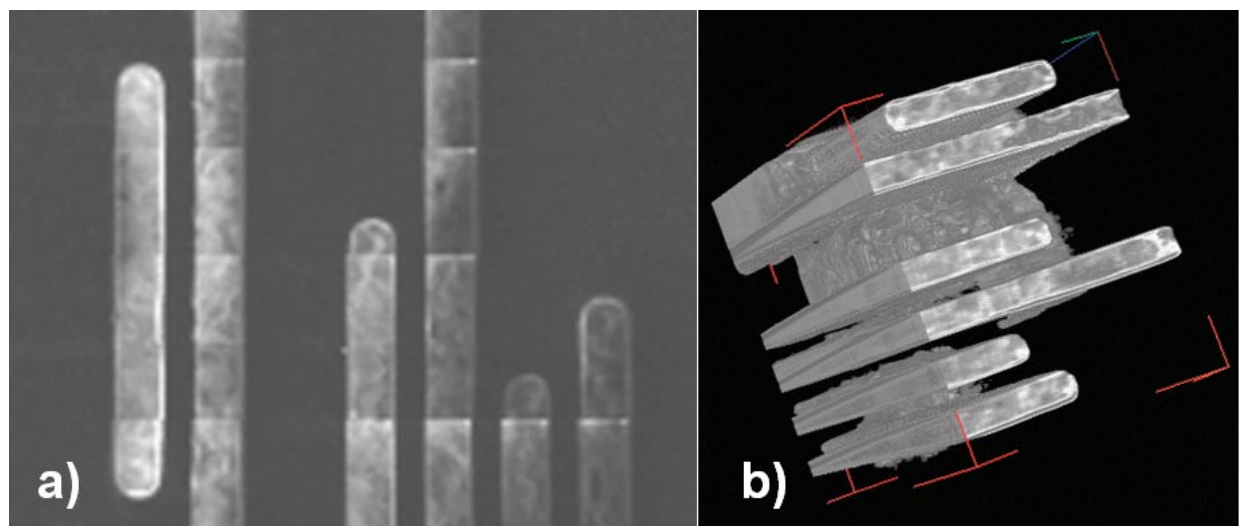

Fig. 6. Top layer of a chip with metal lines $(2 \mu \mathrm{m})$ a) ion scanned and simultaneously imaged and b) 3D reconstruction of metallization down to a total depth of $1.5 \mu \mathrm{m}$ with a layer depth of $3 \mathrm{~nm}$ each
Typical TEM lamellae can nowadays be fabricated straightforwardly with automated routines (Fig. 7a) which handle the preparation from the $\mathrm{Pt}$ or $\mathrm{W}$ protection layer to the fine polishing or even the 'undercut' used to remove the lamella. Such routines also handle thermal or mechanical drift corrections by image recognition. The steps include

i) milling of trenches on each side of the lamella with a high ion current of typically $7 \mathrm{nA}$ and removal of redeposited material;

ii) thinning of the lamella (300 pA), and

iii) final polishing $(100 \mathrm{pA})$. Often,

iv) an additional cleaning step with lowenergy ions ( $7 \mathrm{keV}$ down to $500 \mathrm{eV}$ ) is performed to reduce the amorphized layer (see chapter on artifacts below) on the cross section.

Fig. $7 \mathrm{a}$ and $7 \mathrm{~b}$ illustrate the handling of TEM lamellae with glass needles (ex situ lift out) and a lamella on a $\mathrm{Cu}$ grid with a thin C-film, respectively.

Dual-beam FIBs in particular are helpful when the material samples require special treatments. Four examples are given in Fig. 8. Target preparation [8], i.e. lamellae containing a particular feature at a well-defined location, requires delicate additional steps from the operator. As an example Fig. 8a shows a breakdown site of a transistor gate oxide (TEM image). Fissured materials, shown here on the example of diamond embedded in aluminum (Fig. 8b), are tricky and care has to be taken not to touch one of the columns or gas needles during preparation. Another challenge is materials with intrinsic strain which tend to bend during preparation (Fig. 8c). For lamellae in porous samples one thins the lamella down to a moderate thickness and then fills the pores with supporting material. Inside the FIB this could be deposited W (Fig. 8d), while the material choice increases if the sample is treated outside the FIB chamber. After filling the pores, the lamella is finally polished down to the required thickness. 


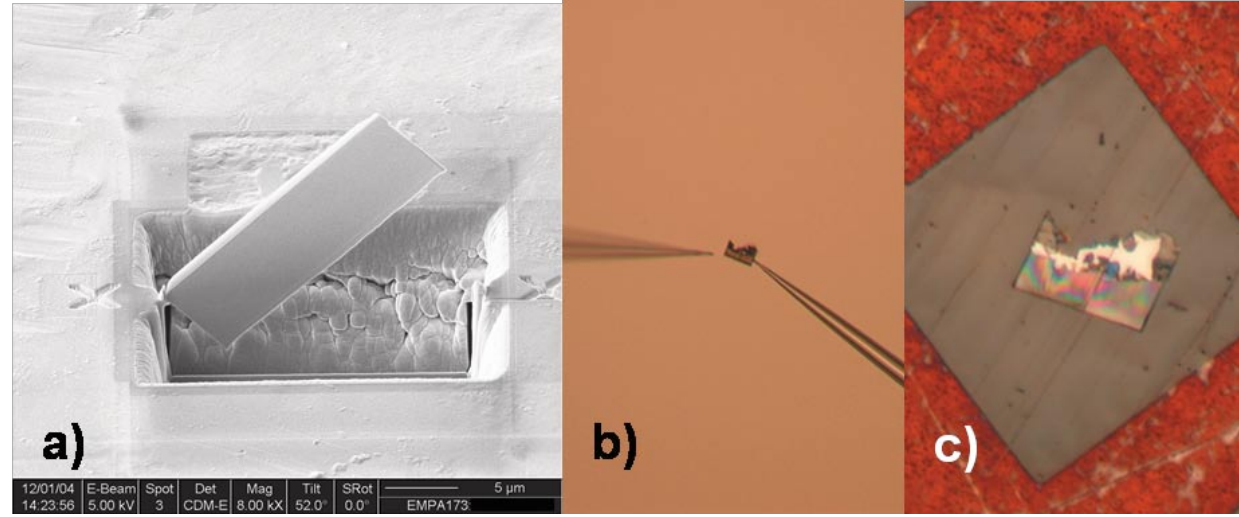

Fig. 7. TEM lamella a) before lift-out (crosses for drift correction), b) lamella manipulation on glass needle tips, and c) lamella on C-film of a Cu-grid

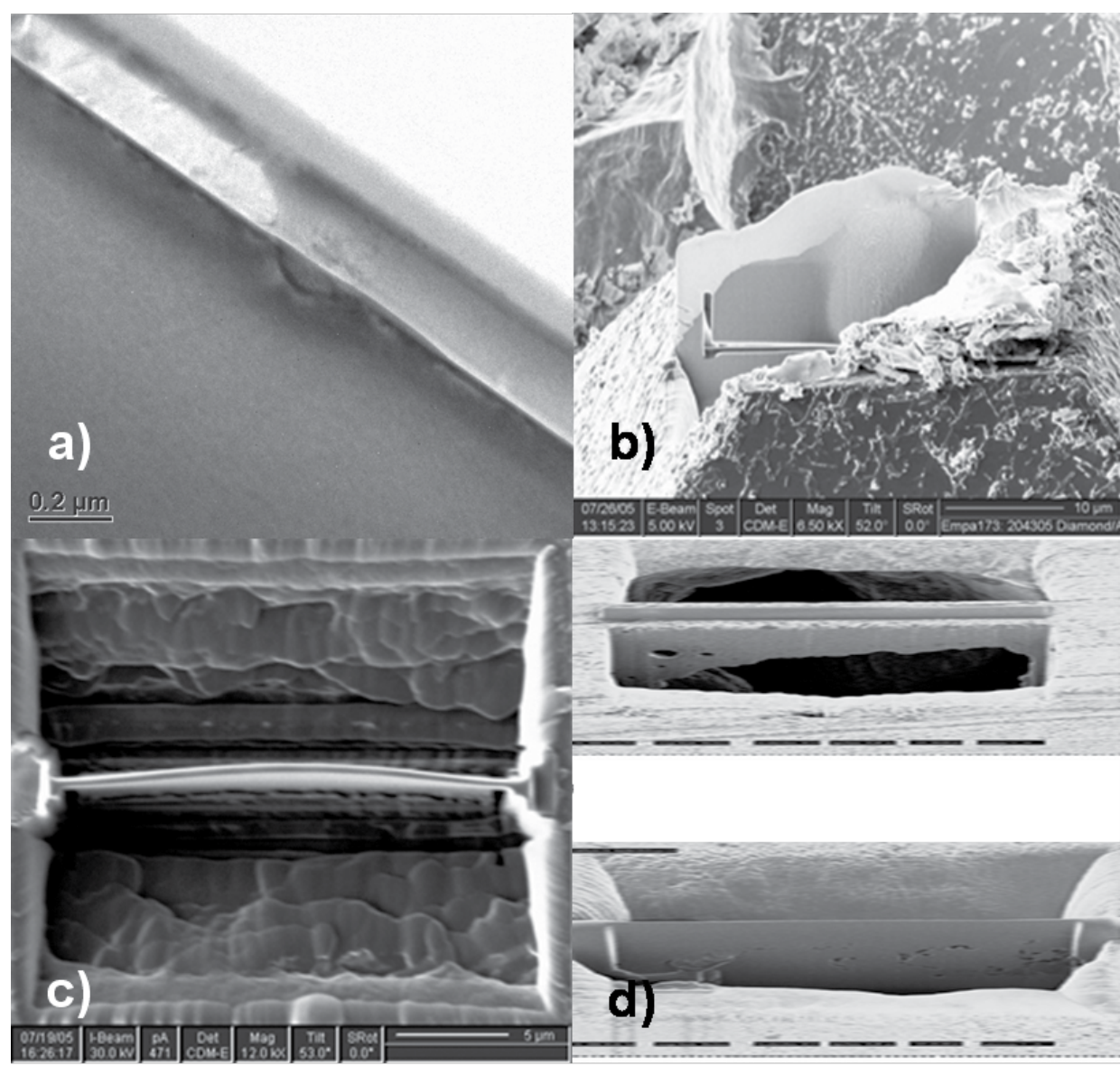

Fig. 8. TEM lamellae: a) target preparation of gate oxide $(5 \mathrm{~nm})$ breakdown site, b) lamella of fissured material, c) lamella of material with intrinsic stress, d) porous lamella (above) filled with $W$ to stabilize before fine polishing (below)

Organic samples like bitumen or polymers and biological samples often require preparation under cryogenic conditions.

\subsection{Pitfalls and Artifacts}

Care has to be taken to avoid pitfalls and to minimize artifacts. During any FIB preparation many disturbing preparation artifacts [30] can occur. Some common ones are described below.

Insulating materials like glass may charge and image distortion and milling at the wrong positions may occur. A charged deposition. Side-wall amorphization can be minimized by proper ion current adjustments during polishing and cleaning. Redeposited material, consisting of sputtered sample material and implanted Ga ions, may cover cross sections, lamellas, and other structures, thereby reducing contrast or even change completely the geometry of the structure to be milled. For TEM lamellae and pillars proper polishing and low voltage cleaning steps can minimize this effect [32]. For three-dimensional prototype structuring scan strategies and dwell times have to be optimized [10]. Scan optimization is also required when self-focusing effect should be avoided, i.e. the deep milling of narrow structures due to reflection of ions at the side-walls and milling further downwards. Fig. 9b shows a Pt-filled cross section of a series of single line scans from left to right. The amorphous redeposited material is clearly visible (grey) and the self-focusing effect can be seen. A top view of a single-scan milled rectangle reveals the three-dimensional behavior of redeposition (Fig. 9c). When fluence is large enough and solubility of $\mathrm{Ga}$ in the sample material is low, Ga precipitation may occur in form of spheres/droplets up to a few $100 \mathrm{~nm}$ in diameter [33] due to mobilization of $\mathrm{Ga}$, diffusion, and phase separation (Fig. 9d). Again, cleaning and polishing steps may help. Sometimes inhomogeneous and rough surfaces are induced in composite materials due to different sputter yields of the composites. For side-walls this effect is known as the curtaining effect. Intrinsic or induced stress in material may lead to bending or moving of thin structures, requiring a very smooth and manually performed preparation. During selective or gas enhanced etching the so-called overspray effect may affect or damage an area not actually irradiated by the ion beam just by the flow of the sometimes aggressive gases on areas sputtered by the beam skirts (Fig. 9e). Even spontaneous reactions may occur. For example, InP with removed native oxide reacts with iodine flowing over the surface. This leads to islands of $\mathrm{InI}_{3}$ (Fig. 9f) which was confirmed by X-ray photoelectron spectroscopy (XPS).

glass surface is shown in Fig. 9a. At very low ion doses $\left(<10^{16} / \mathrm{cm}^{2}\right)$ Si sample swelling [31] up to a few nanometers is observed before actual milling occurs. Surface damage due to lattice distortion and amorphization cannot completely be avoided. However, prior to the treatment of sensitive surfaces first an electron beam induced deposition of metal layers prevents any deterioration or formation of artifacts in the topmost surface layers. After a thin electron deposited Pt layer the remaining layer $(1-4 \mu \mathrm{m})$ will be built up by the faster ion induced

\subsection{Preparation of Structures for Mechanical, Electrical, Thermal, and Magnetic Characterizations}

Newer preparative processes use the 3D structuring capability of FIB-sputtering. To achieve structures of high precision, geometric modeling [10][34] of the sputtering process including redeposition and self-focusing, is required. Otherwise prototyping MEMS, micro-lenses, scanning probe tips and others are difficult to achieve. Certain structures, however, can be produced by innovative scanning strategies [10]. 




Fig. 9. Artifacts: a) sample charging, b) amorphization, redeposition, self-focusing, c) redeposition after milling a rectangular shape, d) Ga contamination, precipitation, e) overspray, f) spontaneous chemical reaction building islands (I with $\operatorname{lnP}$ to $\operatorname{lnl}{ }_{3}$ )

We focus here first on examples of structures prepared for further characterization of mechanical, electrical, and thermal properties in the micro- and nano-regime and second on the prototyping and shaping of tools used for indentation tests as well as improved (higher resolution) scanning probe tips.

Amongst others, micro- and nano-pillars for mechanical characterization by compressive tests in the nanometer-regime can be produced with high accuracy. A first description of micro-pillar fabrication with FIB was given in [35] of fabricating pillars down to a diameter of $5 \mu \mathrm{m}$. We use a preparation which allows us to fabricate pillars down to $50 \mathrm{~nm}$ in diameter and to minimize artifacts as discussed above. First a rough ring of an inner diameter of $2 \mu \mathrm{m}$ and an outer diameter of $7.5 \mu \mathrm{m}$ was milled out of the sample with a current of $3 \mathrm{nA}$ and a beam overlap of $50 \%$; the sample surface hereby is perpendicular to the ion beam. This step takes $100 \mathrm{~s}$ in GaAs. A rough preform of a pillar remains. Then smaller rings were milled concentric to the preform with decreasing diameters and ion currents down to the desired pillar diameter. Each ring does a better polishing and cleaning of the sidewalls of the pillars and reduces the dimension to the final diameter. The final milling was done in concentric rings from the outer radius inwards with the direction changing from clockwise to counterclockwise, each turn with an ion current of $30 \mathrm{pA}$. Milling towards the center avoids redeposition of material on the sidewalls of the pillars. The GaAs pillar in Fig. 10a shows Ga droplets formed by selective sputtering of arsenic due to the local energy injection and transfer of momentum by the gallium ions and further agglomeration due to enhanced diffusion of the remaining species. Also some inhomogeneous artifacts caused by high current preform milling are vis- ible. However, the pillar itself has a very smooth surface due to the optimized cleaning process described above. This can also be seen in the close-up of the pillar, here shown after the compressive test (Fig. 10b). The mechanical characterizations of different semiconductor materials and the change of the mechanical parameters due to downsizing effects will be described elsewhere.

It is suggested that such pillars could also be used as samples for high-resolution TEM-tomography, especially when an additional low-voltage cleaning step is used

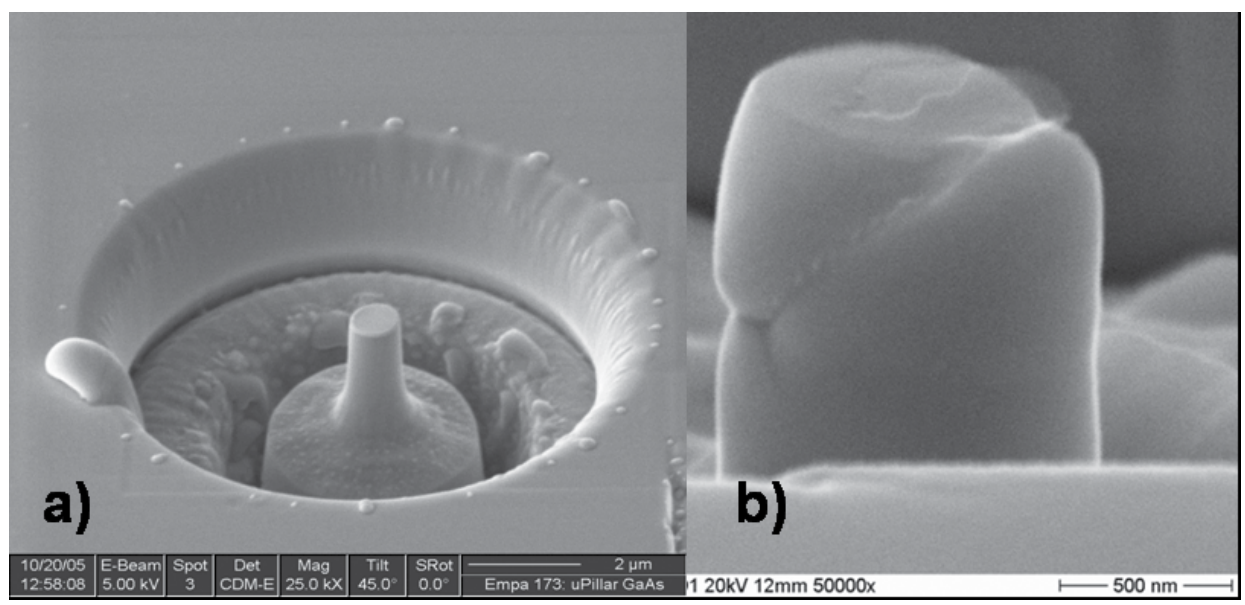

Fig. 10. FIB-fabricated GaAs pillar a) before and b) after compressive test 
to remove remaining amorphous layers on the sidewalls.

For deformation-tracking in micro/nano-mechanical tests like static creep tests, fine periodic line- and dot structures (either deposited or sputtered) can be used. As an example, Figs. 11a and 11b show the deformation of a shallow periodic line structure milled in $\mathrm{Sn}_{3.8} \mathrm{Ag}_{0.7} \mathrm{Cu}$ before and after static creep tests at $80^{\circ} \mathrm{C}$ and $10 \mathrm{MPa}$ shearing strain. It has been shown that in thin $\operatorname{SnAg}_{3.8} \mathrm{Cu}_{0.7}$ solder samples strain concentration in shear bands is the dominant mechanism of fatigue damage [36].

Two- and four-probe electrical measurements for conductivity are well-known techniques. For reliable measurements of individual nano-wires (e.g. $\mathrm{SnO}_{2}$ ) or carbon nanotubes the challenge lies in first contacting them and second determining the contact resistance. With the help of electron or ion assisted chemical deposition of platinum droplets, reproducible contacts could be fabricated and impedance spectroscopy could be applied. Fig. 12 shows an image of a carbon nano-wire with $\mathrm{Pt}$ contacts directly on four metal electrodes. Using the 3- $\omega$ method, based on the fact that the third harmonic amplitude and phase as a response to applied alternate current at fundamental frequency $\omega$ can be expressed in terms of thermal conductivity and diffusivity, the thermal conductivity of individual multiwalled carbon nanotubes was measured. The thermal conductivity was measured at room temperature under vacuum to be $300 \pm 20 \mathrm{~W} / \mathrm{mK}$ [19].

In addition to preparing samples for later analysis, FIB is also used to shape and form tools used to perform analytical tasks, e.g. scanning-probe microscope tips. An example is the sharpening of an AFM-tip for improved resolution, shown in Figs. 13a-c before and after the FIB procedure.

Ultrasoft cantilevers with high force sensitivity have been used to characterize small magnetic particles [37]. An increase in sensitivity is expected, if magnetic probes for MFM are precisely shaped to a defined form to achieve high probe field strength. Fig. 14 illustrates a magnetic CoSm particle attached to a cantilever before and after shaping. The best tip so far had a magnetic moment of $2.9 \cdot 10^{-13} \mathrm{Am}^{2}$ parallel to the cantilever and the coercive field of the tip was $2.2 \mathrm{~T}$, thus close to the bulk value of CoSm. Care has to be taken to have only one grain inside the finished tip.

\section{Conclusions}

For specific nanoanalysis tasks, the appropriate preparative methods which can be performed by focused ion beam instruments were discussed. Starting from typical examples like cross-sections and standard

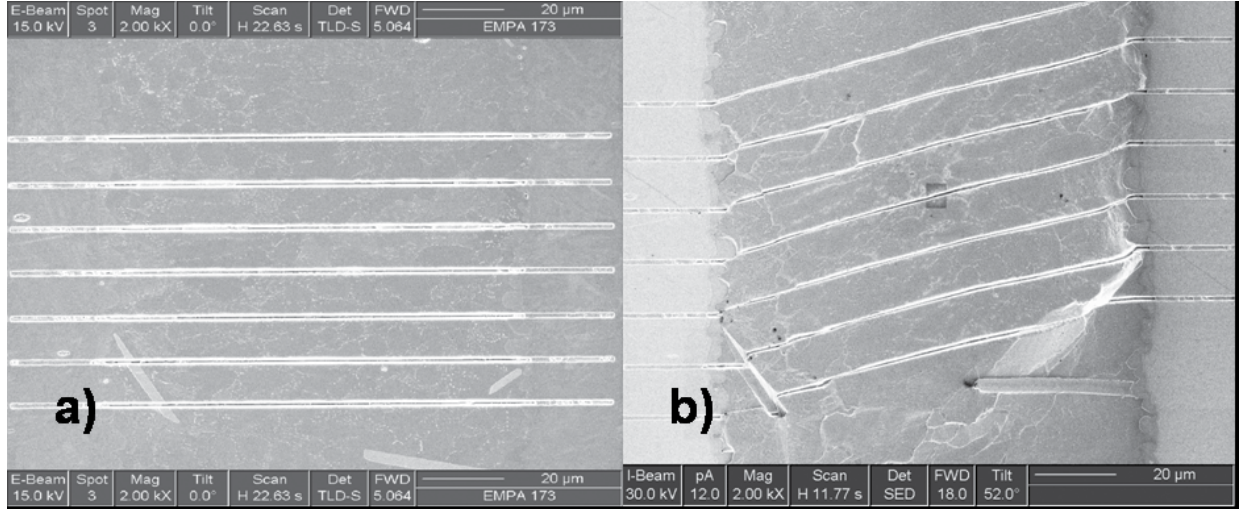

Fig. 11. Periodic line structure in $\mathrm{Sn}_{3.8} \mathrm{Ag}_{0.7} \mathrm{Cu}$ a) before and $\mathrm{b}$ ) after static creep test

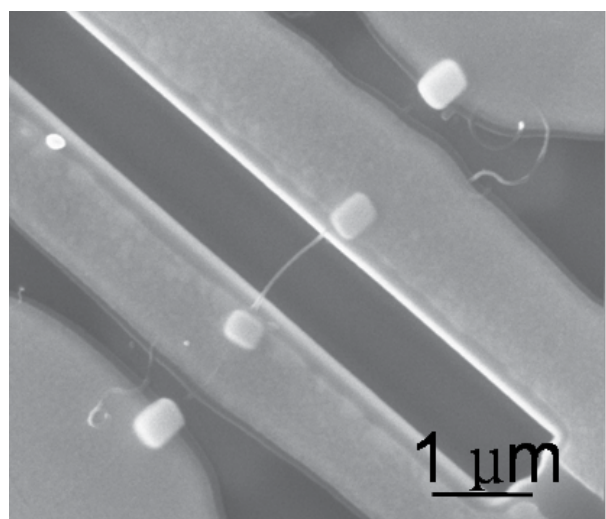

Fig. 12.: Four point contact of carbon nanotube with Pt deposited dots prepared for electrical resistivity and thermal conductivity measurements

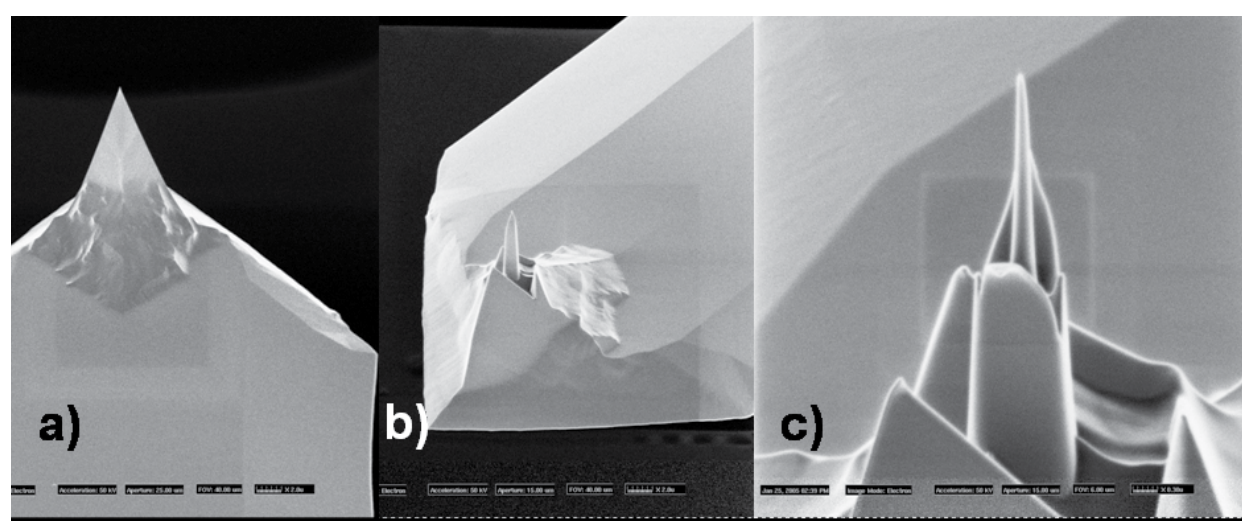

Fig. 13. Atomic force microscope tip a) before and b), c) after sharpening

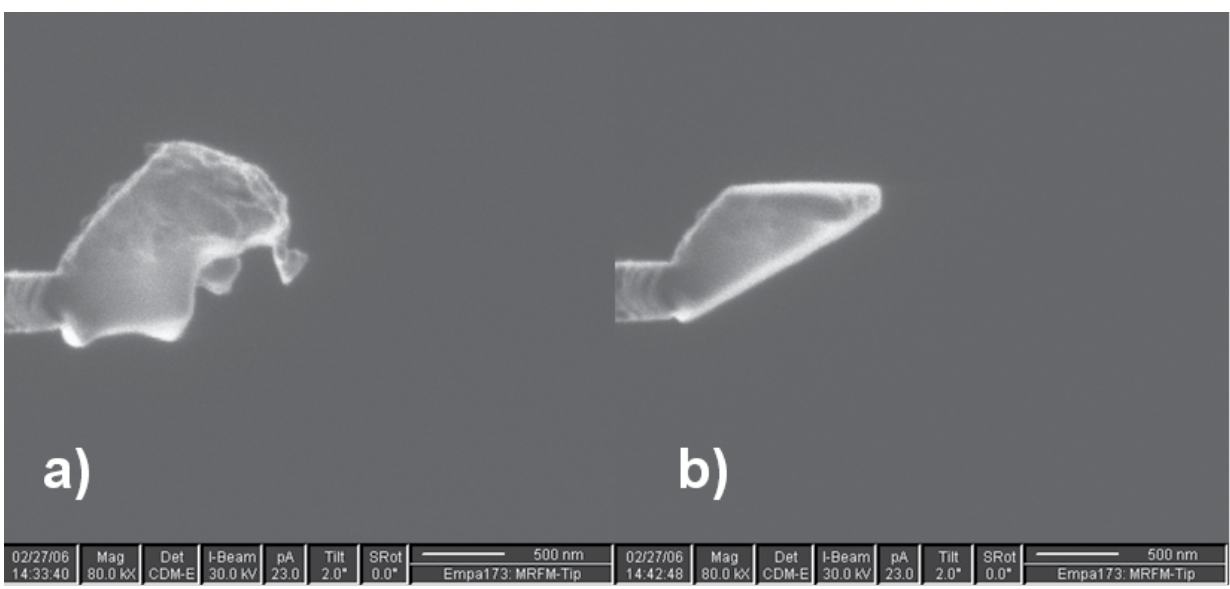

Fig. 14. Shaping of a magnetic CoSm particle as an optimized magnetic force microscope tip a) before and b) after FIB shaping 
TEM-lamella, newer techniques were outlined like FIB-tomography during which milling and imaging is simultaneously performed, or TEM-lamella preparation of porous or fissured materials. Pitfalls and artifacts were illustrated with samples from literature or own samples like, e.g. the spontaneous reaction of InP with $\mathrm{I}_{2}$.

It was shown that the trend in methodology goes towards preparation techniques for the investigation of mechanical, electrical, thermal, and magnetic properties. A new pillar fabrication procedure was discussed with which pillars down to a diameter of $50 \mathrm{~nm}$ are possible. Also, a method was presented to track local deformation from shear or strain by marking the specimen with periodic line- or dot-patterns. Nanowire contacts were demonstrated which allow thermal and electrical characterization without destroying the nano-wires during contacting. Examples of shaping and forming of scanning probe microscope tips were shown, where either sophisticated ion beam scanning strategies or even geometric modeling of structure fabrication with the ion beam are required.

\section{Acknowledgements}

The authors gratefully acknowledge P. Jud, S. Meier, J. Michler, J. Reiner, S. Rast, P. Ruff, C. Soltmann, and J. Tharian for providing images.

Received: September 4, 2006

[1] J. Orloff, M. Utlaut, L. Swanson, 'High resolution focus ion beams', Kluwer, New York, ISBN-0-306-47350-X, 2003.

[2] L.A. Giannuzi, F.A. Stevie, 'Introduction to focused ion beams (instrumentation, techniques, and practice)', Springer Science+Business Media, Inc., 2005.

[3] A.A. Tseng, Small 2005, 1, 924.

[4] R.M. Langford, A.K. Petford-Long, M. Rommeswinkle, S. Egelkamp, Mat. Sci. Techn. 2002, 18, 743.

[5] S. Reyntjens, R. Puers, J. Micromech. Microeng. 2001, 11, 287.

[6] T. Ishitani, K. Umemura, T. Ohnishi, T. Yaguchi, T. Kamino, J. Elect. Microsc. 2004, 53, 443.

[7] R.M. Langford, A.K. Petford-Long, J. Vac. Sci. Technol. 2001, A 19, 2186.
[8] J.C. Reiner, P. Gasser, U. Sennhauser, Microel. Reliability 2002, 42, 1753.

[9] J. Gierak, E. Bourhis, M.N. Mérat Combes, Y. Chriqui, I. Sagnes, D. Mailly, P. Hawkes, R. Jede, L. Bruchhaus, L. Bardotti, B. Prével, A. Hannour, P. Mélinon, A. Perez, J. Ferré, J.-P. Jamet, A. Mougin, C. Chappert, V. Mathet, Microel. Eng. 2005, 78-79, 266

[10] P.M. Nellen, V. Callegari, R. Bronnimann, Microel. Eng. 2006, 83, 1805.

[11] P.P. Jud, P.M. Nellen, U. Sennhauser, $A d v$. Eng. Mat. 2005, 7, 384.

[12] R.J. Fasching, Y. Tao, F.B. Prinz, Sens. Act. B 2005, 108, 964.

[13] G. Villanuevaa, J.A. Plaza, A. SanchezAmores, J. Bausells, E. Martinez, J. Samitier, A. Errachid, Mat. Sci. Eng. 2006, C 26, 164.

[14] C. Menozzi, G.C. Gazzadi, A. Alessandrini, P. Facci, Ultramicroscopy 2005, 104, 220.

[15] M.R. Koblischka, U. Hartmann, T. Sulzbach, J. Magnet. Magn. Mat. 2004, 272 276, 2138

[16] R.J. Fasching, S.J. Bai, T. Fabian, F.B. Prinz, Microel. Eng. 2006, 83, 1638.

[17] C. Lehrer, L. Frey, S. Petersen, T. Sulzbach, O. Ohlsson, T. Dziomba, H.U. Danzebrink, H. Ryssel, Microel. Eng. 2001, 57-58, 721.

[18] A. Vila, F. Hernandez-Ramirez, J. Rodriguez, O. Casals, A. Romano-Rodriguez, J.R. Morante, M. Abid, Mat. Sci. Eng. 2006, C 26, 1063 .

[19] T.Y. Choi, D. Poulikakos, J. Tharian, U. Sennhauser, Appl. Phys. Lett. 2005, 87, 013108.

[20] P. Kitslaar, M. Strassner, I. Sagnes, E. Bourhis, X. Lafosse, C. Ulysse, C. David, R. Jede, L. Bruchhaus, J. Gierak, Microel Eng. 2006, 83, 811.

[21] P. Sigmund, in 'Inelastic Ion-Surface Collisions', Ed. N.H. Tolk, Academic Press, New York, 1977.

[22] J.W. Ward, R.L. Kubena, M.W. Utlaut, J. Vac. Sci. Technol. 1988, B6, 2090.

[23] M. Marko, C. Hsieh, W. Moberlychan, C.A. Mannella, J. Frank, J. Microsc 2006, 222, 42 .

[24] D. Drobne, M. Milani, A. Zrimec, V. Leser, M. B. Zrimec, J. Microsc. 2005, 219 , 29.
[25] B.J. Inkson, M. Mulvihill, G. Mobus, Scripta Mater. 2001, 45, 753.

[26] D.N. Dunn, G.J. Shiflet, R. Hull, Rev. Sci. Instr. 2002, 73, 330.

[27] M.H.F. Overwijk, F.C. Vandenheuvel, C.W.T. Bullelieuwma, J. Vac. Sci. Technol. 1993, B 11, 2021.

[28] L.A. Giannuzzi, F.A. Stevie, Micron 1999, 30, 197.

[29] J. Li, T. Malis, S. Dionne, Mat. Charact. 2006, 57, 64.

[30] B.I. Prenitzer, C.A. Urbanik-Shannon, L.A. Giannuzzi, S.R. Brown, R.B. Irwin, T.L. Shofner, F.A. Stevie, Microsc. Microanal. 2003, 9, 216.

[31] A. Lugstein, B. Basnar, G. Hobler, E. Bertagnolli, J. Appl. Phys. 2002, 92, 4037.

[32] A. Barna, B. Pecz, M. Menyhard, Micron 1999, 30, 267.

[33] J. Reiner, P.M. Nellen, U. Sennhauser, Microel. Reliability 2004, 44, 1583.

[34] E. Platzgummer, A. Biedermann, H. Langfischer, S. Eder-Kapl, M. Kuemmel, S. Cernusca, H. Loeschner, C. Lehrer, L. Frey, A. Lugstein, E. Bertagnolli, Microel. Eng. 2006, 83, 936

[35] M.D. Uchic, D.M. Dimiduk, Mat. Sci. Eng. A 2005, (400-401), 268.

[36] P.P. Jud, G. Grossmann, U. Sennhauser, P.J. Uggowitzer, Adv. Eng. Mat. 2006, 8, 179.

[37] S. Rast, U. Gysin, P. Ruff, C. Gerber, E. Meyer, D.W. Lee, Nanotech. 2006, 17, S189. 